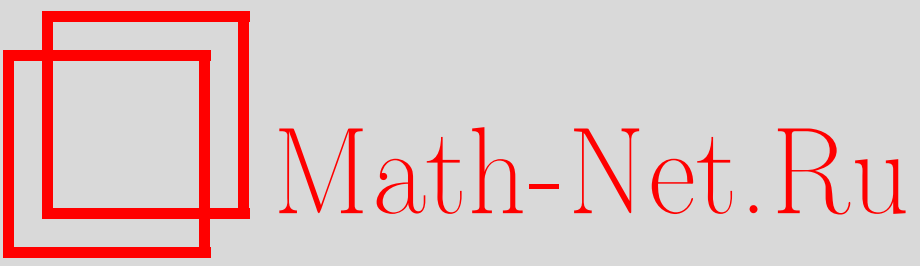

В. В. Терновский, А. М. Хапаев, М. М. Хапаев, Резонансные эффекты в поле стоячей электромагнитной волны и постоянного магнитного поля, ТМФ, 2005, том 142, номер 1, 64-71

DOI: https://doi.org/10.4213/tmf1769

Использование Общероссийского математического портала Math-Net.Ru подразумевает, что вы прочитали и согласны с пользовательским соглашением

http: //www . mathnet.ru/rus/agreement

Параметры загрузки:

IP : 54.147 .182 .235

26 апреля 2023 г., 10:50:59 
ТЕОРЕТИЧЕСКАЯ

И МАТЕМАТИЧЕСКАЯ

ФИЗИКА

Том 142, № 1

январь, 2005

(C) 2005 г. $\quad$ В.В. Терновский*, А. М. Хапаев*, М.М. Хапаев*

\section{РЕЗОНАНСНЫЕ ЭФФЕКТЫ В ПОЛЕ СТОЯЧЕЙ ЭЛЕКТРОМАГНИТНОЙ ВОЛНЫ И ПОСТОЯННОГО МАГНИТНОГО ПОЛЯ}

В статье анализируется взаимодействие релятивистской заряженной частицы с электромагнитным полем, являющимся суперпозицией стоячей волны и постоянного магнитного поля. Показатель преломления среды отличен от единицы. Исследование проводилось в приближении малого сигнала. Для тестирования результатов использовалось компьютерное моделирование исходной системы с учетом двухмасштабности скорости изменения переменных.

Ключевые слова: релятивизм, коэффициент преломления, резонанс.

Проблема взаимодействия заряженных частиц с электромагнитными полями представляет значительный интерес для современной физики элементарных частиц. Движение релятивистских частиц и вопросы излучения изучались для бегуших волн различной поляризации. При этом взаимодействие с интенсивными полями обычно рассматривалось в рамках квантовой электродинамики [1]. Классическое описание процессов обмена энергии релятивистской частицы с волной возможно провести в приближении малой амплитуды электромагнитной волны, когда допустимо пренебречь процессами излучения. В настояшей работе исследуются эффекты взаимодействия заряженных частиц с полем стоячей волны, являюшейся суперпозицией двух встречных бегуших волн.

1. Постановка задачи, интегралы движения. Пусть заряженная частица взаимодействует с полем стоячей электромагнитной волны с вектор-потенциалом

$$
\vec{A}=A[(\sin (\omega \xi)+\sin (\omega \eta)) \vec{i}-(\cos (\omega \xi)+\cos (\omega \eta)) \vec{j}], \quad A=-\frac{c E_{0}}{\omega}
$$

где $c$ - скорость света, $\omega$ - частота волны, $E_{0}$ - амплитуда электрического поля, $\xi$ и $\eta-$ аргументы прямой и вторичной волны:

$$
\xi=t-\frac{z}{c \beta_{\mathrm{f}}}, \quad \eta=t+\frac{z}{c \beta_{\mathrm{f}}}, \quad \beta_{\mathrm{f}}=\frac{1}{k} .
$$

* Московский государственный университет, Москва, Россия. E-mail: vladimir1961@hotmail.com 
Здесь $\beta_{\mathrm{f}}$ - фазовая скорость волны, $k$-показатель преломления. В дальнейшем будем писать вектор-потенциал (1) в виде

$$
\vec{A}=2 A \cos \frac{\omega z}{c \beta_{\mathrm{f}}}[\sin (\omega t) \vec{i}-\cos (\omega t) \vec{j}]
$$

Вектор напряженности магнитного поля $\vec{H}=H_{0} \vec{n}$ направлен вдоль оси $z$.

Классическая ковариантная формулировка уравнений движения Максвелла-Лоренца в электромагнитном поле с вектор-потенциалом $A_{\mu}$ и интеграл энергии-импульса образуют замкнутую систему нелинейных дифференциальных уравнений [2]:

$$
\ddot{x}_{\mu}=\frac{e}{m c}\left(\frac{\partial A_{\mu}}{\partial x_{\nu}}-\frac{\partial A_{\nu}}{\partial x_{\mu}}\right) \dot{x}_{\nu}, \quad \dot{x}_{\mu}^{2}=-c^{2} .
$$

Здесь $m$ - масса, $e$ - заряд частицы, точка означает дифференцирование по собственному времени $\tau$, связанному с лабораторным временем $t$ известным соотношением

$$
\gamma=\frac{d t}{d \tau}=\frac{1}{\sqrt{1-\beta^{2}}}, \quad \beta^{2}=\beta_{\perp}^{2}+\beta_{\|}^{2}, \quad \beta_{\perp}^{2}=\left(\frac{1}{c} \frac{d x}{d t}\right)^{2}+\left(\frac{1}{c} \frac{d y}{d t}\right)^{2}, \quad \beta_{\|}=\frac{1}{c} \frac{d z}{d t},
$$

где $\gamma$ - безразмерная энергия заряда.

Представляется возможным для дальнейшего анализа сформулировать систему (3) в безразмерных переменных:

$$
\begin{gathered}
\varepsilon=\frac{e E_{0}}{m \omega c}, \quad \omega_{0}=\frac{e H_{0}}{m c}, \quad \mu=\frac{\omega_{0}}{\omega}, \quad t=\omega t, \quad \tau=\omega \tau, \\
x=\frac{\omega x}{c}, \quad z=\frac{\omega z}{c}, \quad \dot{x}=\frac{\dot{x}}{c}, \quad \dot{y}=\frac{\dot{y}}{c},
\end{gathered}
$$

$\varepsilon$ - амплитуда волны, $\omega_{0}$ - частота ларморовского врашения электрона в магнитном поле величины $H_{0}$. Ларморовская частота $\omega_{0}$ и частота электромагнитного поля волны $\omega$ предполагаются величинами одного порядка, поэтому $\mu=O(1)$. Безразмерный параметр $\varepsilon$ предполагается малым $(0<\varepsilon \ll 1)$, что означает малость амплитуды плоской волны $E_{0}$. При этом возможно периодическое движение, которое переходит в хаотическое при больших $E_{0}$. Смысл остальных переменных следует из обозначений.

Первые два уравнения системы (3) после подстановки вектор-потенциалов электромагнитного поля (1) и стационарного магнитного $H_{0}$ приводятся к виду

$$
\begin{aligned}
& \ddot{X}=\mu \dot{Y}-\frac{d}{d \tau}(2 \varepsilon \cos (k z) \sin t), \\
& \ddot{Y}=-\mu \dot{X}+\frac{d}{d \tau}(2 \varepsilon \cos (k z) \cos t) .
\end{aligned}
$$

Симметрия полей (1), (2) позволяет понизить порядок уравнений:

$$
\begin{aligned}
\dot{X} & =\mu Y-2 \varepsilon \cos (k z) \sin t+c_{x}, \\
\dot{Y} & =-\mu X+2 \varepsilon \cos (k z) \cos t+c_{y} .
\end{aligned}
$$

3 Теоретическая и математическая физика, т. 142, № 1,2005 г. 
Константы интегрирования, возникающие при переходе к уравнениям первого порядка по $\tau$, определяются соотношениями

$$
\begin{aligned}
& c_{x}=\dot{X}_{0}-\mu Y_{0}+2 \varepsilon \cos z_{0} \sin t_{0}, \\
& c_{y}=\dot{Y}_{0}+\mu X_{0}-2 \varepsilon \cos z_{0} \cos t_{0} .
\end{aligned}
$$

Замена переменных типа сдвига, как и в статье [3]:

$$
X=x-\frac{c_{y}}{\mu}, \quad Y=y+\frac{c_{x}}{\mu},
$$

приводит систему (3) к следующей скалярной системе четырех уравнений:

$$
\begin{aligned}
& \dot{x}=\mu y-2 \varepsilon \cos (k z) \sin t, \\
& \dot{y}=-\mu x+2 \varepsilon \cos (k z) \cos t, \\
& \ddot{z}=-2 \varepsilon \mu k \sin (k z)(y \sin t+x \cos t)+2 \varepsilon^{2} k \sin (2 z), \\
& \dot{\gamma}=-2 \varepsilon \mu \cos (k z)(y \cos t-x \sin t) .
\end{aligned}
$$

Дальнейшее преобразование системы (4) приводит к интегралу движения, связьвающему энергию и поперечные компоненты координат заряда, аналогично сделанному в статье [3]:

$$
\gamma=-\frac{1}{2} \mu\left(x^{2}+y^{2}\right)+R
$$

где $R$ - константа интегрирования, определяемая начальными условиями.

Симметрия электромагнитных полей позволяет перейти к цилиндрической системе координат

$$
x=r \cos \varphi, \quad y=r \sin \varphi, \quad z=z, \quad \theta=t-\varphi
$$

и получить систему, содержащую быстрые и медленные переменные [4], [5], где $r$ и $\dot{z}-$ медленные переменные, их производные $\sim O(\varepsilon), \theta$ и $z$ - быстрые переменные, их производные $\sim O(1):$

$$
\begin{aligned}
\dot{r} & =-\varepsilon[\sin (\theta+k z)+\sin (-k z+\theta)] \\
\dot{\theta} & =\mu+\gamma-\frac{\varepsilon}{r}[\cos (\theta+k z)+\cos (-k z+\theta)] \\
\ddot{z} & =-\varepsilon \mu k r[\sin (\theta+k z)+\sin (-k z+\theta)]+2 \varepsilon^{2} k \sin (2 z), \\
\gamma & =-\frac{1}{2} \mu r^{2}+R
\end{aligned}
$$

Разделение системы на быстрые и медленные переменные связано с выбором параметров электромагнитного поля: амплитуды высокочастотного электромагнитного поля $E_{0}$, его частоты $\omega$, амплитуды стационарного магнитного поля $H_{0}$, ларморовской частоты $\omega_{0}$. При этом, как говорилось, $0<\varepsilon \ll 1, \omega_{0} / \omega \sim O(1)$. При других условиях возможно только хаотическое движение, и такого деления не будет. 
2. Резонансные соотношения. Используя теорию возмушений, получим формальное асимптотическое разложение по степеням $\varepsilon$ задачи (6):

$$
\begin{aligned}
& r=r_{0}+\varepsilon r_{1}(\tau)+\varepsilon^{2} r_{2}(\tau)+\cdots \\
& \theta=\left(\mu+\gamma_{0}\right) \tau+\varepsilon \theta_{1}(\tau)-\varphi+\varepsilon^{2} \theta_{2}(\tau)+\cdots, \\
& z=\dot{z}_{0} \tau+\varepsilon z_{1}(\tau)+\varepsilon^{2} z_{2}(\tau)+\cdots
\end{aligned}
$$

Для энергии $\gamma$ в первом приближении имеем

$$
\begin{gathered}
\gamma=-\varepsilon \mu r_{0}\left[\frac{\cos \left(\sigma_{+} \tau-\varphi_{0}\right)}{\sigma_{+}}-\frac{\cos \left(\sigma_{-} \tau+\varphi_{0}\right)}{\sigma_{-}}\right]+\varepsilon^{2}(\ldots)+\cdots+\text { const } \\
\sigma_{+}=\gamma_{0}+\mu+k \dot{z}_{0}, \quad \sigma_{-}=-\gamma_{0}-\mu+k \dot{z}_{0} .
\end{gathered}
$$

Аналогично выписываются формулы для $z$ и $\theta$. Таким образом, изменение энергии с точностью до $\varepsilon$ происходит по двухпериодическому закону с периодами $T_{+}=2 \pi / \sigma_{+}$, $T_{-}=2 \pi / \sigma_{-}$. Очевидно, если частоты $\sigma_{+} \approx 0$ или $\sigma_{-} \approx 0$, то асимптотическое разложение (7) будет расходиться. Это известная в механике проблема малых знаменателей. Классические методы теории возмущений не позволяют описывать резонансные режимы, поэтому для дальнейшего анализа введем следуюшие понятия.

Пусть $\sigma=-\gamma(\tau)-\mu+k \dot{z}(\tau)$ - комбинационная частота, обычно называемая расстройкой, $\psi=k z(\tau)-\theta(\tau)-$ комбинационная фаза. Резонансом условимся называть такое соотношение параметров, при котором величина расстройки мала на асимптотически большом отрезке времени $\tau \sim 1 / \varepsilon$.

В комбинационную частоту $\sigma$ (расстройку) через $\gamma(\tau)$ входит ларморовский радиус, а следовательно, и формируюшее его внешнее поле $H_{0}$, скорость продольного движения $\dot{z}$, а также коэффициент преломления $k$. Таким образом, все физические величины, характеризуюшие процесс, оказываются связанными резонансными соотношениями. Если $\sigma$ сохраняется вблизи нуля, она представляет баланс скоростей продольного и ларморовского движения. Как известно из эксперимента, если такого баланса нет, то движение становится хаотическим. В правых частях задачи (6) имеются слагаемые с малыми (резонансными) частотами и быстроосциллирующие слагаемые $\cos (k z+\theta)$, $\sin (k z+\theta), \sin (2 k z)$. Согласно принципу усреднения [5] решение системы с некоторыми начальными условиями близко на асимптотически большом отрезке времени к решению усредненной системы с теми же начальными условиями; в частности, к решению системы, в правых частях которой опушены быстроосциллируюшие слагаемые.

Выясним вопрос устойчивости резонанса, используя метод исследования на устойчивость, основанньй на объединении второго метода Ляпунова и метода усреднения и ориентированный на исследование многочастотных систем (см. монографию [4], гл. 4). В теоремах об устойчивости таких систем вводится условие изолированности резонанса, т.е. при удалении траектории от точки резонанса резонансное условие перестает действовать, и возможно оценить снизу резонансную частоту. В системе (6) медленные переменные $-r$ и $\dot{z}$, быстрые $-\theta$ и $z$. Если усреднить (6) по быстрым переменным, исчезнут 
слагаемые $\cos (k z+\theta), \sin (k z+\theta), \sin (2 k z) ;$ положение резонанса $\sigma=0$. Новая система для исследования резонанса имеет вид

$$
\begin{aligned}
\dot{r} & =\varepsilon \sin \psi, \\
\dot{\sigma} & =\varepsilon \mu q \sin \psi, \\
r \dot{\psi} & =r \sigma+\varepsilon \cos \psi, \\
q \gamma & =-\sigma+\text { const, } \quad q=1-k^{2} .
\end{aligned}
$$

Изучим устойчивость и изолированность резонанса $\sigma=0$. В качестве функции Ляпунова выберем величину $|\sigma|$. Зададим $\eta>0,0<\eta_{1}<\eta$ и рассмотрим кольцевую область, состоящую из $\left[-\eta,-\eta_{1}\right],\left[\eta_{1}, \eta\right]$. Устойчивость имеет место, если при начальных значениях расстройки $\sigma_{0}$, удовлетворяюшей условию $\left|\sigma_{0}\right|<\eta_{1}$, для последуюших времен (на бесконечном или асимптотически большом отрезке) справедливо неравенство $|\sigma|<\eta$. Пока справедливо неравенство $|\sigma|<\eta_{1}$, тем более справедливо неравенство $|\sigma|<\eta$. Когда же $|\sigma|$ превысит $\eta_{1}$, для $\sigma$ как комбинационной частоты будет справедлива оценка снизу $|\sigma|>\eta_{1}$, и в системе (8) возможно усреднение по $\psi[4]$, которое дает нуль справа. Это означает, что $\sigma$ сохраняется на асимптотически большом отрезке $\tau \approx 1 / \varepsilon$, и система работает в резонансном режиме. Заметим, что для условий вакуума $\left(k=\beta_{\mathrm{f}}=1\right)$ утверждение очевидно, т.е. в этом случае расстройка - быстроосциллирующая функция с нулевым средним.

Следует подчеркнуть, что усредненная система (8) аналитически интегрируема и сводится к нелинейному уравнению второго порядка для расстройки

$$
(\ddot{\sigma}-a)^{2}=\sigma^{2}\left(2 a\left(\sigma-\sigma_{0}+\frac{1}{2} \mu q r_{0}^{2}\right)-\dot{\sigma}^{2}\right),
$$

где $a=\mu \varepsilon^{2} q$. Аналитическое решение (9) неоднозначно:

$$
\begin{gathered}
\sigma_{1}=\sigma_{0}-\frac{1}{2} \mu q r_{0}^{2}+\frac{1}{2} a\left(\tau^{2}-2 c \tau+c_{0}^{2}\right), \\
\tau+c_{2}= \pm \int_{0}^{\sigma_{2}(\tau)} \frac{d \xi}{\sqrt{-\xi^{4}+8 a\left(\xi-\sigma_{0}+\mu q r_{0}^{2} / 2\right) 2 \xi^{2} \sqrt{-c_{1}}+c_{1}}},
\end{gathered}
$$

где $c_{1}, c_{2}, c_{3}$ - константы интегрирования.

Так как резонанс устойчив по времени, то первое неограниченное решение $\sigma_{1}$ нереализуемо. Второе решение $\sigma_{2}(\tau)$ описывает эллиптические колебания. Случай второго резонанса с расстройкой $\sigma(\tau)=k \dot{z}+\gamma+\mu$ исследуется аналогично. Заметим, что те же резонансные явления имеют место и в лабораторной системе координат. Действительно, переход от $\tau$ к $t$ связан с релятивистским множителем $\gamma: d t=\gamma d \tau$, а $\gamma$ отличается от нуля.

3. Численное моделирование полученных результатов. Проведенное аналитическое исследование процесса взаимодействия релятивистского заряда со стоячей 


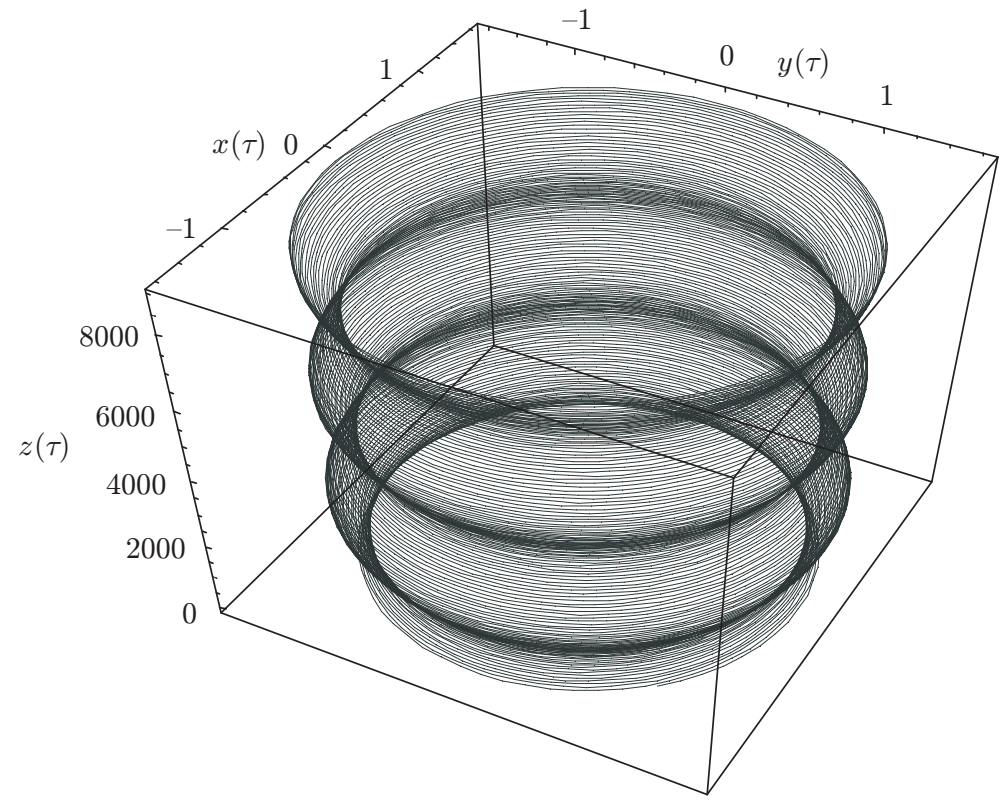

Рис. 1. Периодические траектории релятивистской частицы при резонансе $\beta_{x 0}=\beta_{y 0}=$ $0.1, \beta_{z 0}=0.95, \varepsilon=0.001, \mu=-0.34$ в зависимости от собственного времени $\tau$.

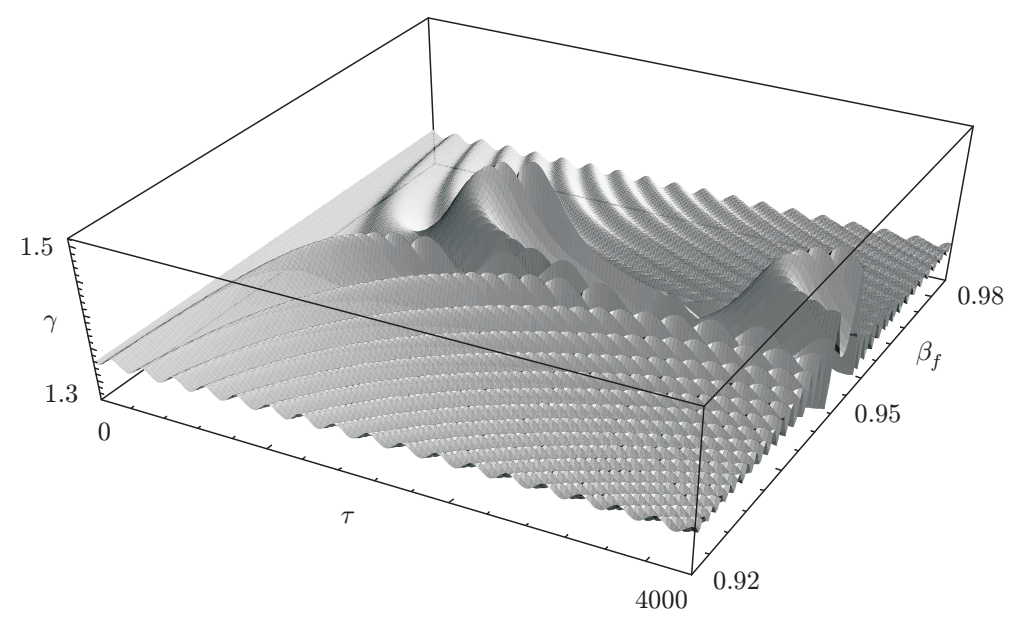

Рис. 2. Колебания энергии $\gamma$ в окрестности резонанса $\beta_{\mathrm{f}}=0.95$ при $\beta_{x 0}=\beta_{y 0}=0.1$, $\beta_{z 0}=0.7, \varepsilon=0.001, \mu=-0.38$ в зависимости от собственного времени $\tau$.

электромагнитной волной (1), как и ожидалось, оказалось чрезвычайно сложной задачей. В конечном итоге удалось установить сушествование параметров, при которых возможны резонансные режимы взаимодействия, и, главное, определить их устойчивость.

Энергетические состояния заряда и соответствующие им траектории приведены на рис. 1-4. Численное моделирование проводилось различными многошаговыми метода- 


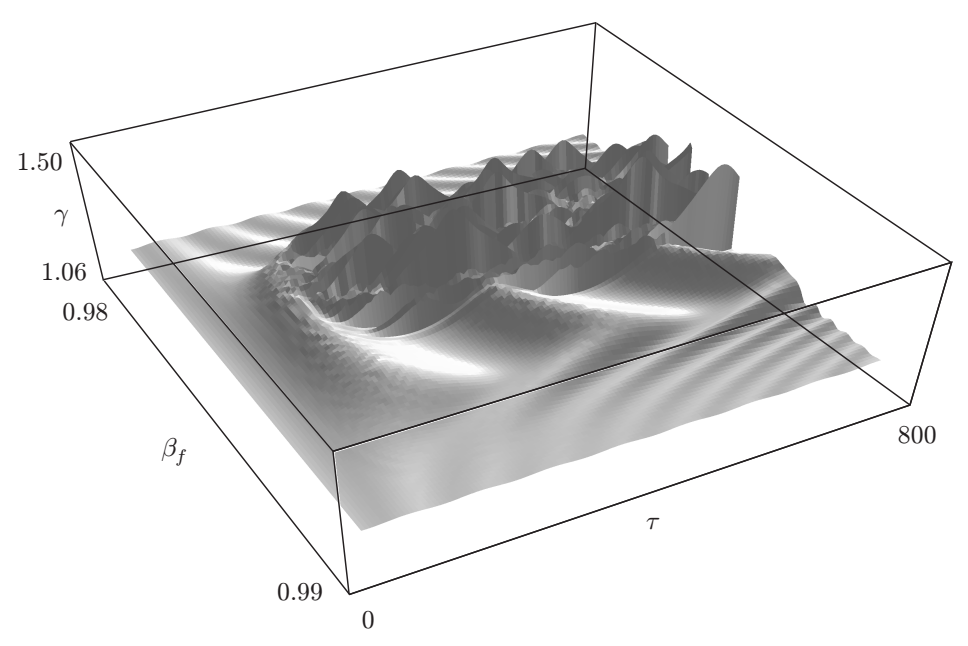

Рис. 3. Возникновение непериодических режимов энергии $\gamma$ при $\beta_{x 0}=\beta_{y 0}=0.1, \beta_{z 0}=$ $0.9, \varepsilon=0.008, \mu=-4.59$ в зависимости от собственного времени $\tau$.

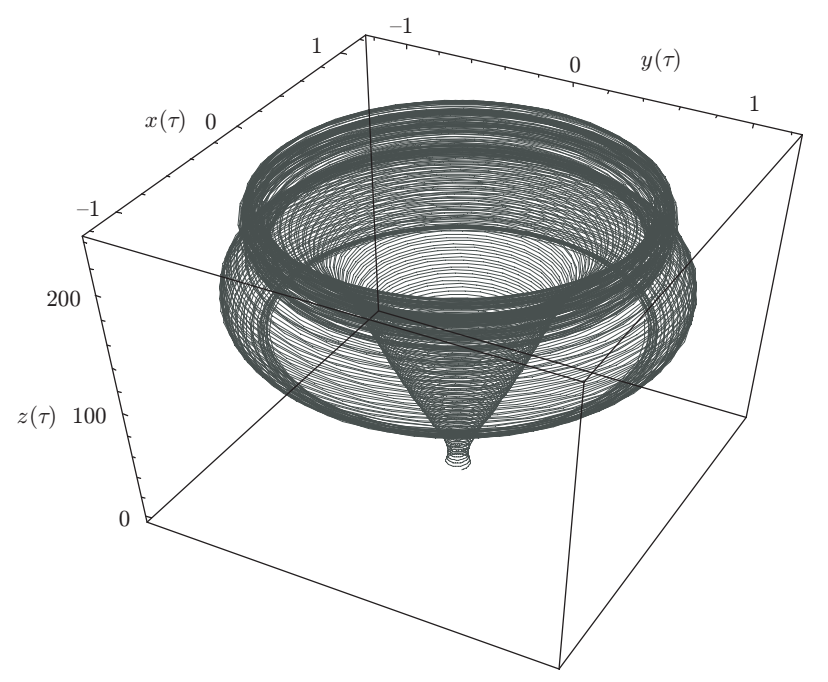

Рис. 4. Непериодические траектории релятивистской частицы при $\beta_{\mathrm{f}}=1.03, \beta_{x 0}=$ $\beta_{y 0}=0.1, \beta_{z 0}=0.9, \varepsilon=0.008, \mu=-4.59$ в зависимости от собственного времени $\tau$.

ми интегрирования нелинейных дифференциальных уравнений системы (6) $\bar{c}$ контролем точности сохранения интеграла движения (5). Так как расчет хаотических режимов движения (рис. 3,4$)$ приводит к жесткому ограничению для шага интегрирования, то применялся метод Isode из пакета Maple фирмы Waterloo Maple Inc.

При выборе параметров задачи, удовлетворяющих условию существования резо- 
нансного взаимодействия, $\beta_{\mathrm{f}}=0.95$ (рис. 1,2 ), численное моделирование изменения энергии $\gamma$ как функции от $\omega \tau$ показывает увеличение амплитуды и периода колебаний энергии в окрестности резонанса по сравнению с нерезонансным режимом (рис. 2). Причем движение происходит по гофрированной цилиндрической поверхности (рис. 1), по оси $z$ отложена длина пролетного пространства в длинах волн.

Как удалось установить, система (6) отличается наличием непериодических решений при $\varepsilon \geqslant 10^{-3}$, что показано на рис. 3,4 . При этом движение происходит в замкнутом пространстве, а непериодический режим означает нерегулярное заполнение траекториями цилиндрической поверхности, изображенной на рис. 4.

4. Заключение. Так как резонансы устойчивы, на практике возможно обеспечить длительное взаимодействие релятивистского пучка заряженных частиц в поле стоячей волны. Однако возникновение хаотических режимов разрушает резонансное состояние и ограничивает применение стоячих волн большой амплитуды. В конфигурациях "продольное магнитное поле и бегушая электромагнитная волна" резонансы отсутствуют, и хаотических траекторий не наблюдается [3], [4]. Проведенный анализ может быть применен для системы взаимодействуюших частищ и бегуших волн различной напряженности, в том числе переменной амплитуды.

Благодарности. Авторы благодарят В. Н. Родионова и В. Р. Халилова за полезное обсуждение работы и ценные замечания.

\section{Список литературы}

[1] И. М. Тернов, В.Р. Халилов, В. Н. Родионов. Взаимодействие заряженных частиц с сильным электромагнитным полем. М.: МГУ, 1982.

[2] Л. Д. Ландау, Е. М. Лифииц. Теория поля. М.: Наука, 1973.

[3] В. В. Терновский, А. М. Хапаев. Фунд. прикл. матем. 2002. Т. 8. № 2. С. 547.

[4] M. М. Хапаев. Усреднение в теории устойчивости. М.: Наука, 1986.

[5] Н. Н. Боголюбов, А.М. Митропольский. Асимптотические методы в теории нелинейных колебаний. М.: Наука, 1974. 